\title{
MODIFIED RIEMANN-LIOUVILLE INTEGRO-DIFFERENTIAL OPERATORS IN THE CLASS OF HARMONIC FUNCTIONS AND THEIR APPLICATIONS
}

\section{B.T. TOREBEK}

\begin{abstract}
In this work we study the properties of some modified Riemann-Liouville integro-differential operators. As an application of the properties of these operators, we consider some local and nonlocal boundary value problems for Laplace equation in a ball. We prove existence and uniqueness for the studied problems. These problems generalize known Dirichlet and Bitsadze-Samarski problems.
\end{abstract}

Keywords: Laplace equation, harmonic function, Bavrin operator, Riemann-Liouville operators, nonlocal problems

Mathematics Subject Classification: 35K20, 35R11

\section{INTRODUCTION}

Let $\Omega=\left\{x \in R^{n}:|x|<1\right\}$ be the unit ball, $n \geqslant 2, \partial \Omega=\left\{x \in R^{n}:|x|=1\right\}$ be the unit sphere. Let $u(x)$ be a harmonic function in domain $\Omega, r=|x|, \theta=\frac{x}{|x|}$. The operators

$$
\begin{gathered}
I^{\alpha}[u](x)=\frac{1}{\Gamma(\alpha)} \int_{0}^{r}(r-\tau)^{\alpha-1} u(\tau \theta) d \tau, \\
D^{\alpha}[u](x)=\frac{d}{d r} I^{1-\alpha}[u](x), 0<\alpha<1,
\end{gathered}
$$

where $\frac{\partial}{\partial r}=\frac{1}{|x|} \sum_{j=1}^{n} x_{j} \frac{\partial}{\partial x_{j}}$ are called the operators of integration and differentiation of order $\alpha>0$ in the sense of Riemann-Liouville [1]. Since $I^{\alpha}[u](x) \rightarrow u(x)$ a.e. as $\alpha \rightarrow 0$ (see [1]), we can let $I^{0}[u](x)=u(x)$. Then $D^{1}[u](x)=\frac{d u}{d r}(x)$.

In work [2], there were introduced the following operators

$$
B^{\alpha}[u](x)=r^{\alpha} D^{\alpha}[u](x), \quad 0<\alpha<1,
$$

in the class of harmonic functions. In works by I.I. Bavrin [4], there were studied the properties of operators

$$
\begin{aligned}
& \delta_{\mu_{1}}=r \frac{d}{d r}+\mu_{1}, \\
& \delta_{\mu_{1}}^{m}=\left(r \frac{d}{d r}+\mu_{1}\right)\left(r \frac{d}{d r}+\mu_{2}\right) \cdot \ldots \cdot\left(r \frac{d}{d r}+\mu_{m}\right),
\end{aligned}
$$

B.T. Torebek, Modified Riemann-Liouville integro-Differential operators in the Class of HARMONIC FUNCTIONS AND THEIR APPLICATIONS.

(C) TOREBEK B.T. 2015.

The work is financially supported by grant financing of scientific and technical programs and projects by the Committee of Science of MES RK (project 0819/GF4).

Submitted May 16, 2015. 


$$
\begin{aligned}
& \delta_{\mu_{1}}^{-1}[u](x)=\int_{0}^{1} t^{\mu_{1}-1} u(t x) d t, \\
& \delta_{\mu_{1}}^{-m}[u](x)=\frac{1}{(m-1) !} \int_{0}^{1} t^{\mu_{1}-1}(1-t)^{m-1} u(t x) d t
\end{aligned}
$$

in the class of functions harmonic in a ball, where $\mu_{j}>0, \mu_{j}=\mu_{1}+m-1, j=1,2, \ldots, m$.

Since $(m-1) !=\Gamma(m)$, in view of the construction of operator $\delta_{\mu}^{-m}$ we see that this operator is defined also for the fractional values of parameter $m$. Then a natural question appear on defining the inverse operator for $\delta_{\bar{\mu}}^{-m}$ in the case of fractional values of parameter $m$. Following work [3], we introduce the modification of operator (1)

$$
\delta_{\mu}^{\alpha}[u](x)=r^{-\mu} B^{\alpha}\left[r^{\mu} u\right](x), \quad \mu>0, \quad 0<\alpha<1 .
$$

In the present work we construct fractional analogues of operators $\delta_{\bar{\mu}}^{-m}, \delta_{\bar{\mu}}^{m}$ and we show that the results of work [4] are valid for the general case as well.

Let $u(x)$ be a function harmonic in domain $\Omega, \alpha$ and $\mu$ be nonnegative real numbers and $\mu+1=\mu_{1}$.

We consider the operators

$$
\begin{aligned}
B_{\mu}^{-\alpha}[u](x) & =\left\{\begin{array}{l}
u(x), \quad \alpha=0 \\
\frac{1}{\Gamma(\alpha)} \int_{0}^{1}(1-s)^{\alpha-1} s^{m+\mu-\alpha} u(s x) d s, \quad \alpha>0,
\end{array}\right. \\
B_{\mu}^{\alpha}[u](x) & =\left\{\begin{array}{l}
u(x), \quad \alpha=0, \\
\delta_{\mu}^{\alpha}[u](x), \quad 0<\alpha<1, \\
\delta_{\mu_{1}}^{m}\left[\delta_{\mu}^{\alpha-m}[u]\right](x), \quad m \leqslant \alpha<m+1, \quad m=1,2, \ldots
\end{array}\right.
\end{aligned}
$$

If $\alpha=m$ and $\mu>0$, for (2) we obtain Bavrin operator $B_{\mu_{1}}^{m}=\delta_{\mu_{1}}^{m}$, while if $\mu=0$ and $0<\alpha<1$, then $B_{0}^{\alpha}$ coincides with operator (1).

\section{Properties of OPERATORS $B_{\mu}^{\alpha}$ AND $B_{\mu}^{-\alpha}$}

Let $H_{k}(x)$ be a homogeneous harmonic polynomial of degree $k \in N_{0}=\{0,1, \ldots\}$. Then employing the identities $H_{k}(x)=H_{k}(r \theta)=r^{k} H_{k}(\theta)$, one can prove easily the following lemma.

Lemma 1. Let $\alpha>0, \mu \geqslant 0$ and $H_{k}(x)$ be a homogeneous harmonic polynomial of degree $k \in N_{0}=\{0,1, \ldots\}$. Then the identities

$$
\begin{aligned}
& B_{\mu}^{\alpha}\left[H_{k}\right](x)=\frac{\Gamma(k+m+\mu+1)}{\Gamma(k+m-\alpha+\mu+1)} H_{k}(x), \\
& B_{\mu}^{-\alpha}\left[H_{k}\right](x)=\frac{\Gamma(k+m-\alpha+\mu+1)}{\Gamma(k+m+\mu+1)} H_{k}(x)
\end{aligned}
$$

hold true.

Theorem 1. Let $m \leqslant \alpha<m+1, \mu \geqslant 0$ and a function $u(x)$ be harmonic in $\Omega$. Then the functions $B_{\mu}^{\alpha}[u](x)$ and $B_{\mu}^{-\alpha}[u](x)$ are harmonic in domain $\Omega$.

Proof. Since function $u(x)$ is harmonic in ball $\Omega$, as $|x| \leqslant \rho<1$ it is expanded into the series [5]

$$
u(x)=\sum_{k=0}^{\infty} \sum_{i=1}^{h_{k}} u_{k}^{(i)} H_{k}^{(i)}(x),
$$


where $\left\{H_{k}^{(i)}(x), i=1, \ldots, h_{k}\right\}$ is the complete system of homogeneous harmonic polynomials of degree $k$ and $u_{k}^{(i)}$ are coefficients of expansion $(5)$. Applying formally operator $B_{\mu}^{\alpha}$ to series (5) and taking into consideration identity (3), we obtain

$$
B_{\mu}^{\alpha}[u](x)=\sum_{k=0}^{\infty} \sum_{i=1}^{h_{k}} \frac{\Gamma(k+m+\mu+1)}{\Gamma(k+m-\alpha+\mu+1)} u_{k}^{(i)} H_{k}^{(i)}(x) .
$$

By the asymptotic estimate for Gamma function it is easy to show that

$$
\lim _{k \rightarrow \infty} \sqrt[k]{\frac{\Gamma(k+m+\mu+1)}{\Gamma(k+m-\alpha+\mu+1)}}=1 .
$$

Then the convergence radius of series (6) coincides with the convergence radius of series (5) and thus, its sum is a function harmonic in $\Omega$.

Similarly, employing (4), one can prove that the function $B_{\mu}^{-\alpha}[u](x)$ is harmonic. The proof is complete.

Theorem 2. Let a function $u(x)$ be harmonic in ball $\Omega$. Then for each $x \in \Omega$ the identities

$$
u(x)=\frac{1}{\Gamma(\alpha)} \int_{0}^{1}(1-s)^{\alpha-1} s^{\mu+m-\alpha} B_{\mu}^{\alpha}[u](s x) d s
$$

hold true.

Proof. We represent function $u(x)$ harmonic in ball $\Omega$ as series (5) and transform it as follows:

$$
u(x)=\sum_{k=0}^{\infty} \sum_{i=1}^{h_{k}} \frac{\Gamma(k+m-\alpha+\mu+1)}{\Gamma(k+m+\mu+1)} \frac{\Gamma(k+m+\mu+1)}{\Gamma(k+m-\alpha+\mu+1)} u_{k}^{(i)} H_{k}^{(i)}(x) .
$$

Taking into consideration identities (3), (4) and the uniform convergence of series (8) in $x$ as $|x| \leqslant \rho<1$, we obtain

$$
\begin{aligned}
u(x) & =\sum_{k=0}^{\infty} \sum_{i=1}^{h_{k}} \frac{u_{k}^{(i)}}{\Gamma(\alpha)} \int_{0}^{1}(1-s)^{\alpha-1} s^{\mu+m-\alpha} B_{\mu}^{\alpha}\left[H_{k}^{(i)}\right](s x) d s \\
& =\int_{0}^{1} \frac{(1-s)^{\alpha-1}}{\Gamma(\alpha)} s^{\mu+m-\alpha} B_{\mu}^{\alpha}\left[\sum_{k=0}^{\infty} \sum_{i=1}^{h_{k}} u_{k}^{(i)} H_{k}^{(i)}\right](s x) d s=\int_{0}^{1} \frac{(1-s)^{\alpha-1}}{\Gamma(\alpha)} s^{\mu+m-\alpha} B_{\mu}^{\alpha}[u](s x) d s .
\end{aligned}
$$

The proof is complete.

Theorem 3. Let function $u(x)$ be harmonic in domain $\Omega$ and $B_{\mu}^{\alpha}[u](x) \in C(\bar{\Omega})$. Then for each $m \leqslant \beta<\alpha, \mu \geqslant 0$ the derivative $B_{\mu}^{\beta}[u](x)$ exists and the formula

$$
B_{\mu}^{\beta}[u](x)=\frac{1}{\Gamma(\alpha-\beta)} \int_{0}^{1}(1-s)^{\alpha-\beta-1} s^{\mu+m-\alpha} B_{\mu}^{\alpha}[u](s x) d s
$$

holds true.

Proof. Employing representation (6), we rewrite function $B_{\mu}^{\beta}[u](x)$ as follows:

$$
B_{\mu}^{\beta}[u](x)=\sum_{k=0}^{\infty} \sum_{i=1}^{h_{k}} \frac{\Gamma(k+m+\mu+1)}{\Gamma(k+m-\beta+\mu+1)} u_{k}^{(i)} H_{k}^{(i)}(x)
$$




$$
\begin{aligned}
& =\sum_{k=0}^{\infty} \sum_{i=1}^{h_{k}} \frac{\Gamma(k+m+\mu+1)}{\Gamma(k+m-\alpha+\mu+1)} \frac{\Gamma(k+m-\alpha+\mu+1)}{\Gamma(k+m-\beta+\mu+1)} u_{k}^{(i)} H_{k}^{(i)}(x) \\
& =\sum_{k=0}^{\infty} \sum_{i=1}^{h_{k}} \frac{u_{k}^{(i)}}{\Gamma(\alpha-\beta)} \int_{0}^{1}(1-s)^{\alpha-\beta-1} s^{\mu+m-\alpha} B_{\mu}^{\alpha}\left[H_{k}^{(i)}\right](s x) d s \\
& =\frac{1}{\Gamma(\alpha-\beta)} \int_{0}^{1}(1-s)^{\alpha-\beta-1} s^{\mu+m-\alpha} B_{\mu}^{\alpha}[u](s x) d s .
\end{aligned}
$$

The proof is complete.

Theorem 4. Let function $u(x)$ be harmonic in domain $\Omega$. Then for each $x \in \Omega$ the identities

$$
B_{\mu}^{-\alpha}\left[B_{\mu}^{\alpha}[u]\right](x)=B_{\mu}^{\alpha}\left[B_{\mu}^{-\alpha}[u]\right](x)=u(x)
$$

hold true.

Proof. We apply operator $B_{\mu}^{-\alpha}$ to the function $B_{\mu}^{\alpha}[u](x)$. By the definition of operator $B_{\mu}^{-\alpha}$ we have

$$
B_{\mu}^{-\alpha}\left[B_{\mu}^{\alpha}[u]\right](x)=\frac{1}{\Gamma(\alpha)} \int_{0}^{1}(1-s)^{\alpha-1} s^{\mu+m-\alpha} B_{\mu}^{\alpha}[u](s x) d s .
$$

By identity (7) the latter expression is equal to $u(x)$. The first identity in 10 is proven. To the prover the other, we apply operator $B_{\mu}^{\alpha}$ to the function $B_{\mu}^{-\alpha}[u](x)$ :

$$
\begin{aligned}
B_{\mu}^{-\alpha}\left[B_{\mu}^{\alpha}[u]\right](x) & =\frac{1}{\Gamma(\alpha)} B_{\mu}^{\alpha}\left[\int_{0}^{1}(1-s)^{\alpha-1} s^{\mu+m-\alpha} u(s x) d s\right] \\
& =\delta_{\mu_{1}}^{m} \int_{0}^{1} \frac{(1-s)^{\alpha-1}}{\Gamma(\alpha)} \frac{s^{\mu+m-\alpha} r^{\alpha-m-\mu}}{\Gamma(m+1-\alpha)} \frac{d}{d r} \int_{0}^{r}(r-\tau)^{m-\alpha} \tau^{\mu} u(s \tau \theta) d \tau d s .
\end{aligned}
$$

It is easy to make sure that the identities

$$
\begin{aligned}
\frac{r^{\alpha-m-\mu}}{\Gamma(m+1-\alpha)} \frac{d}{d r} \int_{0}^{r}(r-\tau)^{m-\alpha} \tau^{\mu} u(s \tau \theta) d \tau \\
\quad=\frac{r^{\alpha-m-\mu}}{\Gamma(m+1-\alpha)} \frac{d}{d r} \int_{0}^{r t}\left(r-\frac{t}{s}\right)^{m-\alpha}\left(\frac{t}{s}\right)^{\mu} u(t \theta) \frac{d t}{s} \\
=\frac{(r s)^{\alpha-m-\mu}}{\Gamma(m+1-\alpha)} \frac{d}{d(r s)} \int_{0}^{r s}(r s-t)^{m-\alpha} t^{\mu} u(t \theta) d t=B_{\mu}^{\alpha}[u](s x)
\end{aligned}
$$

and $r \frac{d}{d r}=(r s) \frac{d}{d(r s)}$ hold true, where we have taken into consideration that $\theta=\frac{x}{|x|}=\frac{t x}{|t x|}$. Hence, we have

$$
B_{\mu}^{\alpha}\left[B_{\mu}^{-\alpha}[u]\right](x)=\frac{1}{\Gamma(\alpha)} \int_{0}^{1}(1-s)^{\alpha-1} s^{\mu+m-\alpha} B_{\mu}^{\alpha}[u](s x) d s .
$$

The proof is complete.

Thus, it follows from Theorem 4 that operators $B_{\mu}^{\alpha}$ and $B_{\mu}^{-\alpha}$ are mutually inverse in the class of harmonic functions in ball $\Omega$. 


\section{LOCAL PROBLEM}

We consider the following problem.

Problem L. Find a function $u(x)$ harmonic in ball $\Omega$ such that function $B_{\mu}^{\alpha}[u](x)$ is continuous in $\bar{\Omega}$ and satisfies the identity $B_{\mu}^{\alpha}[u](x)=f(x), x \in \partial \Omega$, on sphere $\partial \Omega$.

We note that similar problems with boundary operators of integer high order were considered in works [4], [6]-[9], and fractional order operators were studied in works [2], 10]-[14].

Let $v(x)$ be a classical solution to the Dirichlet problem in ball $\Omega$

$$
\left\{\begin{array}{cl}
\Delta v(x)=0, & x \in \Omega \\
v(x)=f(x), & x \in \partial \Omega
\end{array}\right.
$$

The following statements are true.

Theorem 5. Let $f(x) \in C(\partial \Omega)$. Then problem $L$ is uniquely solvable and is represented as

$$
u(x)=B_{\mu}^{-\alpha}[v](x)
$$

where $v(x)$ solves problem (11).

Proof. Suppose that Problem L is solvable and a solution is $u(x)$. We apply operator $B_{\mu}^{\alpha}$ to $u(x)$ and denote $B_{\mu}^{\alpha}[u](x)=v(x)$. Since $B_{\mu}^{\alpha}[u](x) \in C(\bar{\Omega})$, then $v(x) \in C(\bar{\Omega})$. Since $u(x)$ is harmonic in $\Omega$, in view of Theorem 1 function $v(x)$ is also harmonic in ball $\Omega$ and

$$
\left.v(x)\right|_{\partial \Omega}=\left.B_{\mu}^{\alpha}[u](x)\right|_{\partial \Omega}=f(x) .
$$

Thus, function $v(x)$ solves Dirichlet problem (11). At that, if $f(x) \in C(\partial \Omega)$, then this problem is uniquely solvable and $v(x) \in C(\bar{\Omega})$. We apply operator $B_{\mu}^{-\alpha}$ to the identity $B_{\mu}^{\alpha}[u](x)=v(x)$. Since the integrals

$$
\int_{0}^{1}(1-\tau)^{\alpha-1} \tau^{\mu+m-\alpha} v(\tau x) d \tau
$$

have weak singularities at $\tau=0$ and $\tau=1$ as $\alpha \in[m, m+1), \mu>0, \mu+m-\alpha \neq 0$, such functions are continuous in $x \in \bar{\Omega}$ once $v(x) \in C(\bar{\Omega})$. Hence, we can apply operator $B_{\mu}^{-\alpha}$ to the functions in $C(\bar{\Omega})$. By the first identity in $(10)$ we obtain identity $\sqrt{12}$, i.e.,

$$
B_{\mu}^{-\alpha}[v](x)=B_{\mu}^{-\alpha}\left[B_{\mu}^{\alpha}[u]\right](x)=u(x) .
$$

Vice versa, suppose that function $v(x)$ solves Dirichlet problem $(11)$ for $f(x) \in C(\partial \Omega)$. It is clear that $v(x) \in C(\bar{\Omega})$. We consider function $u(x)=B_{\mu}^{-\alpha}[v](x)$. By the second identity in (10) we shall have

$$
B_{\mu}^{\alpha}[u](x)=B_{\mu}^{\alpha}\left[B_{\mu}^{-\alpha}[v]\right](x)=v(x) .
$$

Therefore, function $u(x)$ is harmonic in $\Omega$ and

$$
\left.B_{\mu}^{\alpha}[u]\right|_{\partial \Omega}=\left.v\right|_{\partial \Omega}=f(x) .
$$

The proof is complete.

\section{NONLOCAL PROBLEM WHEN THE SUPPORT OF DATA DO NOT INTERSECT THE BOUNDARY OF DOMAIN}

In this section we study the solvability of a nonlocal problem with operator $B_{\mu}^{\alpha}$, when the supports of data do not intersect the boundary of domain. Suppose that we are given the sequences of numbers $\delta_{j}$ and $a_{j}, j=1,2, \ldots$, satisfying the conditions

$$
0<\delta_{j} \leqslant b<1,\left.\quad \sum_{j=1}^{\infty}\right|_{j} \mid<\infty .
$$


Consider the following problem.

Problem N. Find a function $u(x) \in^{2}(\Omega) \cap(\bar{\Omega})$ harmonic in ball $\Omega$, for which function $B_{\mu}^{\alpha}[u](x) \in(\bar{\Omega})$ satisfies the condition

$$
B_{\mu}^{\alpha}[u](x)-\sum_{j=1}^{\infty} a_{j} B_{\mu}^{\beta}[u]\left(\delta_{j} x\right)=f(x), \quad x \in \partial \Omega .
$$

Nonlocal boundary value problems are rather interesting generalizations of classical problems appearing at the same time in mathematical models for real processes and phenomena in physics, engineering, sociology, ecology, etc. During several last decades, in mathematical literature there appeared a series of works devoted to nonlocal problems for differential equations. One of the first papers was by A.V. Bitsadze and A.A. Samarskii [15], in which new formulations of problems for elliptic equations were proposed and which served as a starting point for most part of studies in this direction. The considered problem is the simplest generalization of Bitsadze-Samarskii problem for boundary operators of non-integer order. We note that in the case $\alpha=\beta=0$, i.e., as $B_{\mu}^{\alpha}=B_{\mu}^{\beta} \equiv \mathrm{I}$ is the identity mapping, in the one-dimensional case similar problems were studied in work [16], while the case $n \geqslant 2$ was considered in works [17], [18], [19]. We note also that similar nonlocal problems for boundary operators of fractional order were studied in works [2], [20], [21], [22].

To study problem N, we shall make use of the following auxiliary theorem.

Theorem 6. Suppose that $0<\delta_{j} \leqslant b<1, j=1,2, \ldots, f(x) \in C(\partial \Omega)$ and the inequality

$$
\sum_{j=1}^{\infty}\left|a_{j}\right| \leqslant \frac{\Gamma(m-\beta+\mu+1)}{\Gamma(m-\alpha+\mu+1)}
$$

holds true. Then

1) if the condition

$$
\sum_{j=1}^{\infty} a_{j} \neq \frac{\Gamma(m-\beta+\mu+1)}{\Gamma(m-\alpha+\mu+1)}
$$

is satisfied, then the problem

$$
\left\{\begin{array}{l}
\Delta v(x)=0, x \in \Omega \\
v(x)-\sum_{j=1}^{\infty} a_{j} B_{\mu}^{\beta-\alpha}[v]\left(\delta_{j} x\right)=f(x), \quad x \in \partial \Omega
\end{array}\right.
$$

is uniquely solvable;

2) if the condition

$$
\sum_{j=1}^{\infty} a_{j} \equiv \frac{\Gamma(m-\beta+\mu+1)}{\Gamma(m-\alpha+\mu+1)}
$$

holds true, then problem (15) is solvable if and only if

$$
\int_{\partial \Omega} f(x) d s_{x}=0
$$

3) if the solution exists, then under condition (14) it is unique and under condition (16) it is unique up an additive constant.

Proof. Let us study the uniqueness of solution to problem (15). Suppose that function $v(x)$ solves problem (15) as $f(x)=0$. We denote

$$
M=\max _{\partial \Omega}|\mathrm{v}(x)|=\left|\mathrm{v}\left(x_{0}\right)\right|, x_{0} \in \partial \Omega .
$$


If $v(x) \neq$ const, then the maximum principle for harmonic functions we have $|v(x)|<M$ for each $x \in \Omega$. By the assumption of problem (15) and the definition of operator $B_{\mu}^{\beta-\alpha}$ for $f(x)=0$ we obtain

$$
M=\left|\sum_{j=1}^{\infty} a_{j} B_{\mu}^{\beta-\alpha}[v]\left(\delta_{j} x_{0}\right)\right| \leqslant \sum_{j=1}^{\infty} \frac{\left|a_{j}\right|}{\Gamma(\alpha)} \int_{0}^{1}(1-s)^{\alpha-1} s^{\mu+m-\alpha}\left|v\left(s \delta_{j} x_{0}\right)\right| d s .
$$

Since $0<\delta_{j} \leqslant b<1, j=1,2, \ldots$, then $\delta_{j} x_{0} \in \Omega$ and for each $s \in[0,1]$ the points $s \delta_{j} x_{0}$ also belong ball $\Omega$. Hence, for each $s \delta_{j} x_{0} \in \Omega$ we have $\left|v\left(s \delta_{j} x_{0}\right)\right|<M$ and therefore,

$$
M \leqslant \sum_{j=1}^{\infty}\left|a_{j}\right|\left|B_{\mu}^{\beta-\alpha}[v]\left(\delta_{j} x_{0}\right)\right|<M \frac{\Gamma(m-\alpha+\mu+1)}{\Gamma(m-\beta+\mu+1)} \sum_{j=1}^{\infty}\left|a_{j}\right| .
$$

If (13) holds true, we obtain the contradiction $M<M$. Hence, if condition (13) holds true, we necessarily have $v(x)=$ const. Substituting in this case $v(x) \equiv C$ into the formulation of problem (15), we have $C=0$, or identity (16). Thus, under conditions (13) and (14) we get $v(x) \equiv 0$. If (16) holds true, then an arbitrary constant also solves homogeneous problem (15). Indeed, if $v(x) \equiv C$, substituting in into the formulation of problem (15), we have $C-C=0$. The uniqueness is proven.

We proceed to studying the existence of solution to problem $(15)$. We denote by $\mu(x)=$ $\left.v(x)\right|_{\partial \Omega}$ the trace of an unknown harmonic function $v(x)$ on $\partial \Omega$. We seek a solution to problem (15) as the Poisson integral

$$
v(x)=\int_{\partial \Omega} P(x, y) \mu(y) d S_{y},
$$

where $P(x, y)=\frac{1}{\omega_{n}} \frac{1-|x|^{2}}{|x-y|^{n}}$ is the Poisson kernel for Dirichlet problem 11 . Substituting function (18) into the formulation of problem (15), we obtain the following integral equation

$$
\mu(x)-\sum_{j=1}^{\infty} a_{j} \int_{0}^{1} \frac{(1-s)^{\alpha-\beta-1}}{\Gamma(\alpha-\beta)} s^{\mu+m-\alpha}\left[\int_{\partial \Omega} P\left(s \delta_{j} x, y\right) \mu(y) d s_{y}\right] d s=f(x), \quad x \in \partial \Omega,
$$

for unknown function $\mu(x)$.

Switching the integration order in the left hand side of identity (19), we obtain

$$
\mu(x)-\sum_{j=1}^{\infty} a_{j} \int_{\partial \Omega} P_{\alpha, \beta}\left(\delta_{j} x, y\right) \mu(y) d S_{y}=f(x), x \in \partial \Omega,
$$

where

$$
P_{\alpha, \beta}\left(\delta_{j} x, y\right)=\int_{0}^{1} \frac{(1-s)^{\alpha-\beta-1}}{\Gamma(\alpha-\beta)} s^{\mu+m-\alpha} P\left(s \delta_{j} x, y\right) d s .
$$

We denote

$$
K(x, y)=-\sum_{j=1}^{\infty} a_{j} P_{\alpha, \beta}\left(\delta_{j} x, y\right), \quad x, y \in \partial \Omega .
$$

Then equation 190 is represented as

$$
\mu(x)+\int_{\partial \Omega} K(x, y) \mu(y) d S_{y}=f(x), \quad x \in \partial \Omega .
$$

We observe that for each $x, y \in \partial \Omega$ and $0<\delta_{j} \leqslant b<1, j=1,2, \ldots$, the conditions

$$
\left|\delta_{j} x-y\right|=\left|y-\delta_{j} x\right| \geqslant 1-\delta_{j} \geqslant 1-b>0,
$$


and

$$
\left|\delta_{j} x-y\right|^{2}=\left|\delta_{j} y-x\right|^{2}
$$

hold true. Then it is easy to show that by condition (21), kernel $K(x, y)$ is continuous on $\partial \Omega \times \partial \Omega$, and by condition (22) it is symmetric, i.e., $K(x, y)=K(y, x)$. Therefore, Fredholm theorems [23] are applicable to integral equation (20). It implies easily the statement of the theorem. Indeed, if conditions (13) and (14) are satisfied, homogeneous problem (15) and therefore, the homogeneous problem associated with $(20)$ are uniquely solvable. Then by Fredholm theorem [23] integral equation (20) is solvable for each $f(x) \in C(\partial \Omega)$ and therefore, function $(18)$ is the unique solution to problem (15). If conditions (13) and (16) are satisfied, then a constant solves homogeneous problem (15). Therefore, in this case the homogeneous integral equation corresponding to (20) has one linearly independent solution and this solution is the function $\mu(x)=C$, where $C$ is an arbitrary constant. Since $K^{*}(x, y)=K(y, x)=K(x, y)$, the dual equation corresponding to (20) also has the solution $\mu^{*}(x)=C$.

Then by Fredholm theorem, integral equation (20) is solvable if and only if conditions (17) are satisfied. Thus, if condition (17) is satisfied, function (18) is also the solution to problem (15). The proof is complete.

We proceed to the main problem. The following statement holds true.

Theorem 7. Suppose that $0<\delta_{j} \leqslant b<1, j=1,2, \ldots, f(x) \in C(\partial \Omega)$ and inequality (13) holds true. Then

if condition (14) is satisfied, then Problem $N$ is uniquely solvable;

if condition (16) is satisfied, then Problem $N$ is solvable if and only if condition (17) is satisfied; at that, the solution to the problem is unique up an additive constant;

if Problem $N$ is solvable, it is represented as

$$
u(x)=B_{\mu}^{-\alpha}[v](x),
$$

where $v(x)$ solves problem (15).

Proof. Let $u(x)$ be a solution to Problem N. We apply operator $B_{\mu}^{\alpha}$ to function $u(x)$ and denote $B_{\mu}^{\alpha}[u](x)=v(x)$. By Theorem 2 for each $x \in \Omega$ identity (7) is true. Employing formula (9), we have

$$
B_{\mu}^{\beta}[u]\left(\delta_{j} x\right)=B_{\mu}^{\beta-\alpha}[v]\left(\delta_{j} x\right) .
$$

Since $u(x)$ is harmonic in $\Omega$, by Theorem 1 function $v(x)$ is also harmonic in ball $\Omega$ and the assumption of problem (15) are satisfied. Thus, if $u(x)$ solves Problem N, function $v(x)$ solves problem (15). Suppose that conditions $(13)$ and $(14)$ are satisfied. Then by Theorem 6 for each $f(x) \in C(\partial \Omega)$ problem $(15)$ is uniquely solvable and $v(x) \in C(\bar{\Omega})$. If we apply operator $B_{\mu}^{-\alpha}$ to the identity $B_{\mu}^{\alpha}[u](x)=v(x)$ by the first identity, Theorem 4 implies $u(x)=B_{\mu}^{-\alpha}[v](x)$ for each $x \in \Omega$. The harmonicity of this function follows from Theorem 1, while the assumption of problem 15 is checked straightforwardly:

$$
B_{\mu}^{\alpha}[u](x)-\sum_{j=1}^{\infty} a_{j} B_{\mu}^{\beta}[u]\left(\delta_{j} x\right)=v(x)-\sum_{j=1}^{\infty} a_{j} B_{\mu}^{\beta-\alpha}[v]\left(\delta_{j} x\right)=f(x), \quad x \in \partial \Omega .
$$

The first part of the theorem is proved.

Suppose now that condition 16 is satisfied. Then the identity $u(0)=C$ for the function

$$
w(x)=B_{\mu}^{\alpha}[u](x)-\sum_{j=1}^{\infty} a_{j} B_{\mu}^{\beta}[u]\left(\delta_{j} x\right)
$$

we obtain

$$
w(0)=B_{\mu}^{\alpha}[u](0)-\sum_{j=1}^{\infty} a_{j} B_{\mu}^{\beta}[u](0)=0 .
$$


And since function $w(x)$ solves Dirichlet problem (11), under the condition $w(0)=0$ we arrive at necessary condition (17). This, condition (17) is necessary for solvability of problem $\mathrm{N}$ under condition (16). Let us show that condition (17) is necessary and sufficient for the solvability of Problem N under condition (16). By Theorem 6, under conditions (17) and (16), problem (15) is uniquely solvable up to an additive constant and $v(x) \in C(\bar{\Omega})$. Then the function $u(x)=B_{\mu}^{-\alpha}[v](x)$ satisfies all the assumptions of the theorem. The proof is complete.

\section{NONLOCAL PROBLEM WHEN THE SUPPORTS OF DATA INTERSECT WITH THE BOUNDARY OF DOMAIN}

We consider Problem $\mathrm{N}$ when the sequence of numbers $\delta_{j}$ satisfy the conditions: $0<\delta_{j}<1$, $j=1,2, \ldots$, and $\delta_{j} \rightarrow 1$ as $j \rightarrow \infty$. The following statement holds true.

Lemma 2. Let $f(x) \in \partial \Omega$ and

$$
\sum_{j=1}^{\infty} \frac{\left|a_{j}\right|}{\left(1-\delta_{j}\right)^{n-1}}<\infty .
$$

Then

1) if conditions (13)-(14) are satisfied, then problem (15) is uniquely solvable;

2) if conditions (13), (16) are satisfied, then problem (15) is solvable up to an additive constant if and only if condition (17) is satisfied.

Lemma 2 can be proven in the same way as Theorem 6. At that, by conditions (23), the kernel of integral equation (20) is symmetric and bounded.

The main result of this section is the following statement.

Theorem 8. Suppose that $0<\delta_{j}<1, j=1,2, \ldots, \lim _{j \rightarrow \infty} \delta_{j}=1, f(x) \in C(\partial \Omega)$ and 23 is true. Then

1) if conditions (13)-(14) are satisfied, then Problem $N$ is uniquely solvable;

2) if conditions (13), (16) are satisfied, then Problem $N$ is solvable if and only if condition (17) is satisfied; at that, the solution to the problem is unique up to an additive constant;

3) if Problem $N$ is solvable, then its solution is represented as

$$
u(x)=B_{\mu}^{-\alpha}[v](x),
$$

where $v(x)$ solves problem 15).

Proof of Theorem 8 is almost literally reproduces the proof of Theorem 7 , where one should use Lemma 2 instead of Theorem 6 .

\section{EXAMPLES}

Example 1. Let $n=2,0<\alpha<1$ and $\mu=0$. By Theorem 5 the unique solution to Problem $L$ reads as

$$
\begin{aligned}
u(r, \varphi) & =B_{\mu}^{-\alpha}[v](r, \varphi)=\frac{1}{\Gamma(\alpha)} \int_{0}^{1}(1-s)^{\alpha-1} s^{-\alpha} u(r s, \varphi) d s \\
& =\int_{0}^{1} \frac{(1-s)^{\alpha-1}}{\Gamma(\alpha)} \frac{s^{-\alpha}}{2 \pi}\left\{\int_{-\pi}^{\pi} \frac{1-(r s)^{2}}{1-2 r s \cos (\varphi-\theta)+(r s)^{2}} f(\theta) d \theta\right\} d s \\
& =\frac{1}{2 \pi} \int_{-\pi}^{\pi}\left\{\int_{0}^{1} \frac{(1-s)^{\alpha-1}}{\Gamma(\alpha)} \frac{s^{-\alpha}\left(1-(r s)^{2}\right)}{1-2 r s \cos (\varphi-\theta)+(r s)^{2}} d s\right\} f(\theta) d \theta
\end{aligned}
$$


The following identity

$\int_{0}^{1} \frac{(1-s)^{\alpha-1}}{\Gamma(\alpha)} \frac{s^{-\alpha}\left(1-(r s)^{2}\right)}{1-2 r s \cos (\varphi-\theta)+(r s)^{2}} d s=2 \Gamma(1-\alpha)\left(\frac{\cos \left[(1-\alpha) \operatorname{arctg} \frac{r \sin (\varphi-\theta)}{1-r \cos (\varphi-\theta)}\right]}{\left(1-2 r \cos (\varphi-\theta)+r^{2}\right)^{\frac{1-\alpha}{2}}}-\frac{1}{2}\right)$

was proven in [24]. Then finally we obtain

$$
u(x)=\frac{\Gamma(1-\alpha)}{\pi} \int_{-\pi}^{\pi}\left\{\frac{\cos \left[(1-\alpha) \operatorname{arctg} \frac{r \sin (\varphi-\theta)}{1-r \cos (\varphi-\theta)}\right]}{\left(1-2 r \cos (\varphi-\theta)+r^{2}\right)^{\frac{1-\alpha}{2}}}-\frac{1}{2}\right\} f(\theta) d \theta .
$$

Example 2. Let $a_{1} \neq 0$ and $a_{j}=0, j=2,3, \ldots$

We consider a homogeneous harmonic polynomial of degree $p$

$$
u_{p}(x)=\sum_{|\beta|=p} c_{\beta} x_{1}^{\beta_{1}} \cdot x_{2}^{\beta_{2}} \cdot \ldots \cdot x_{n}^{\beta_{n}} .
$$

We consider the action of operator $B_{\mu}^{\alpha}$ on function $u_{p}(x)$. By identity (3),

$$
B_{\mu}^{\alpha}\left[u_{p}\right](x)=\frac{\Gamma(p+m+\mu+1)}{\Gamma(p+m-\alpha+\mu+1)} u_{p}(x) .
$$

Since $u_{p}\left(\delta_{1} x\right)=\delta_{1}^{p} u_{p}(x)$, then

$$
\begin{aligned}
B_{\mu}^{\alpha}\left[u_{p}\right](x)-a_{1} B_{\mu}^{\beta}\left[u_{p}\right]\left(\delta_{1} x\right) & =\frac{\Gamma(p+m+\mu+1)}{\Gamma(p+m-\alpha+\mu+1)} u_{p}(x)-a_{1} \frac{\Gamma(p+m+\mu+1)}{\Gamma(p+m-\beta+\mu+1)} \delta_{1}^{p} u_{p}(x) \\
& =\left(\frac{\Gamma(p+m+\mu+1)}{\Gamma(p+m-\alpha+\mu+1)}-a_{1} \frac{\Gamma(p+m+\mu+1)}{\Gamma(p+m-\beta+\mu+1)} \delta_{1}^{p}\right) u_{p}(x) .
\end{aligned}
$$

Therefore, harmonic polynomial $u_{p}(x)$ is a solution to homogeneous Problem $\mathrm{N}$ corresponding to

$$
a_{1}=\frac{\Gamma(p+m-\beta+\mu+1)}{\Gamma(p+m-\alpha+\mu+1)} \delta_{1}^{-p}>1 .
$$

We also note that for $n=3$, the number of homogeneous harmonic polynomials of degree $p$ is equal to $2 p+1$.

\section{BIBLIOGRAPHY}

1. A.A. Kilbas, H.M. Srivastava, J.J. Trujillo. Theory and applications of fractional differential equations. North-Holland Mathematics Studies, 204. Elsevier, Amsterdam (2006).

2. V.V. Karachik, B.Kh. Turmetov, B.T. Torebek. On some integro-differential operators in the class of harmonic functions and their applications// Matem. Trudy. 14:1, 99-125 (2011). [Siber. Adv. Math. 22:2, 115-134 (2012).]

3. A.A. Kilbas, A.A. Tityura. Fractional derivative of Marsho-Hadamard type and inversion of fractional integrals // Dokl. Nats. Akad. Nauk Belarusi. 50:4, 5-10 (2006). (in Russian).

4. I.I. Bavrin. Operators for harmonic functions and their applications // Differ. Uravn. 21:1, 9-15 (1985). [Diff. Equat. 21:1, 6-10 (1985).]

5. E.M. Stein, G. Weiss. Introduction to Fourier analysis on Euclidean spaces. Princeton Mathematical Series. Princeton University Press, Princeton (1971).

6. I.I. Bavrin. Integro-differential operators for harmonic functions in convex domains and their applications // Differ. Uravn. 24:9, 1629-1631 (1988). (in Russian).

7. V.B. Sokolovskij. On a generalization of the Neumann problem // Differ. Uravn. 24:9, 1629-1631 (1988). (in Russian).

8. A.V. Bitsadze. On the Neumann problem for harmonic functions // Dokl. Akad. Nauk SSSR. 311:1, 11-13 (1990). [Sov. Math. Dokl. 41:2, 193-195 (1990).] 
9. V.V. Karachik, B.Kh. Turmetov. On a problem for the harmonic equation // Izv. Akad. Nauk UzSSR, Ser. Fiz.-Mat. Nauk. 4, 17-21 (1990).

10. B.T. Torebek, B.Kh. Turmetov. On solvability of a boundary value problem for the Poisson equation with the boundary operator of a fractional order // Boundary Value Problems. 2013:93, 18pp. (2013).

11. B.Kh. Turmetov. A boundary value problem for the harmonic equation // Differ. Uravn. 32:8, 1089-1092 (1996). [Differ. Equat. 32:8, 1093-1096 (1996).]

12. B.Kh. Turmetov. On smoothness of a solution to a boundary value problem with fractional-order boundary operator // Matem. Trudy. 7:1, 189-199 (2004). [Siber. Adv. Math. 15:2, 115-125 (2005).]

13. B.Kh. Turmetov, B.T. Torebek. Modified Bavrin operators and their applications // Differ. Uravn. 51:2, 240-250 (2015). [Diff. Equat. 51:2, 243-254 (2015).]

14. A.S. Berdyshev, B.Kh. Turmetov, B.J. Kadirkulov. Some properties and applications of the integrodifferential operators of hadamard-marchaud type in the class of harmonic functions // Sibirsk. Matem. Zhurn. 53:4, 752-764 (2012). [Siber. Math. J. 53:4, 600-610 (2012).]

15. A.V. Bitsadze, A.A. Samarskii. On some simple generalizations of linear elliptic boundary problems // Dokl. Akad. Nauk SSSR. 185:4, 739-740 (1969). [Sov. Math. Dokl. 10, 398-400 (1969).]

16. V.A. Il'in, E.I. Moiseev. A nonlocal boundary value problem for the Sturm-Liouville operator in the differential and difference treatments // Dokl. Akad. Nauk SSSR. 291:3, 534-539 (1986). [Sov. Math. Dokl. 34, 507-511 (1987).]

17. A.K. Pulatov. On a Bitsadze-Samarskij problem // Differ. Uravn. 25:3, 537-540 (1989).

18. F. Criado, F.Jr. Criado, N. Odishelidze. On the solution of some non-local problems // Czechoslovak Math. Jo. 54:2, 487-498 (2004).

19. K.Yu. Kishkis. On a nonlocal problem for functions harmonic in a multiply connected domain // Differ. Uravn. 23:1, 174-177 (1987). (in Russian).

20. A.S. Berdyshev, B.J. Kadirkulov, J.J. Nieto. Solvability of an elliptic partial differential equation with boundary condition involving fractional derivatives // Compl. Variab. Ellipt. Equat. 59:4, 680-692 (2013).

21. M.A. Sadybekov, B.K. Turmetov, B.T. Torebek. Solvability of nonlocal boundary-value problems for the Laplace equation in the ball // Electr. J. Diff. Equat. 2014:157, 1-14 (2014).

22. M. Kirane, B.T. Torebek. On a nonlocal problem for the Laplace equation in the unit ball with fractional boundary conditions // Math. Meth. Appl. Sci. (2015) doi:10.1002/mma.355.

23. A.B. Vasil'eva, N.A. Tikhonov. Integral equations. Fizmatlit, Moscow (2002). (in Russian.)

24. B.T. Torebek, B.Kh. Turmetov. On solvability of some inverse problems for Laplace equation with boundary Riemann-Liouville operator // Vestnik KarGU. Ser. Matem. 69:1, 113-121 (2013). (in Russian).

Berikbol Tillabaiuly Toreber,

Institute of mathematics and mathematical modelling,

Pushkin str. 125,

050010, Almaty, Kazakhstan

Al-Farabi Kazakh National University,

Al-Farabi str. 71,

050040, Almaty, Kazakhstan

E-mail: turebekb85@mail.ru 